\title{
Primary pulmonary MPNST-A rare case report
}

\begin{abstract}
Primary lung sarcoma is an extremely rare tumour accounting for $<0.5 \%$ of all the lung tumours. Here we present a case of primary pulmonary malignant peripheral nerve sheath tumour (MPNST) in a 60 year male patient. The diagnosis was made on histopathological examination (HPE) and confirmed on immunohistochemistry (IHC). Patient underwent surgery and received 2 cycles of post-operative chemotherapy however, expired eight months after diagnosis. We conclude that MPNST should be included in the differential diagnosis of spindle cell tumours of lung.
\end{abstract}

Keywords: MPNST, IHC, lung cancer, sarcoma, primary
Volume 6 Issue 2 - 2018

\author{
Tazeen Jeelani, Suhail Mushtaq, Rumana \\ Makhdoomi
}

Department of pathology, India

\begin{abstract}
Correspondence: Tazeen Jeelani, senior resident department of pathology SKIMS soura Srinagar Jammu and Kashmir, India, Tel +91907012 9836, +91901826 7086,

Email tazeenjeelani@gmail.com
\end{abstract}

Received: April 17,2018 | Published: April 27, 2018

\section{Introduction}

MPNST is one of the rare tumours. Its incidence is 1 per $1,000,000$ and represents about $3 \%$ to $10 \%$ of all soft tissue sarcomas. ${ }^{1}$ MPNST can be seen in any part of the body like head and neck, extremities, trunk or retroperitoneum, ${ }^{2,3}$ but intra-thoracic primary pulmonary MPNST with or without NF-1 is uncommon. Only few adult cases have been reported so far. ${ }^{4,5}$

\section{Case presentation}

A 60 years old smoker, normotensive, non-diabetic presented with chief complaints of cough, fever and weight loss for 20 days. On examination the patient was conscious, co-operative, well oriented, with a respiratory rate of $18 / \mathrm{min}$, blood pressure of $120 / 70$ and pulse rate of $86 / \mathrm{min}$. Cardiovascular and per-abdomen examination were clinically normal. However, on auscultation decreased breath sounds were found on left lower side of chest. Chest radiograph revealed a lesion in the left lung. CT scan (contrast enhanced) of chest was done which showed a $71 \times 59 \mathrm{~mm}$ cystic lesion in the superior segment of left lower lobe. Cyst showed internal septations without calcification. USG abdomen and PFT (pulmonary function tests) were normal. Hydrated serology was negative and not suggestive. Routine complete blood counts, LFT (liver function test) and KFT (kidney function tests), were within normal limits. The patient underwent left postero-lateral thoracotomy with lower lobe lobectomy. Intra-operatively there was large bronchogenic mass occupying almost whole of the left lobe with multiple hilar nodes. On gross examination, we received a lobe of lung measuring $15 \times 9.5 \times 5 \mathrm{~cm}$. Serial slicing of the lung showed a well circumscribed mass measuring $7 \times 8 \mathrm{~cm}$. Cut section showed variegated appearance with extensive hemorrhagic and necrotic areas. On light microscopy a spindle cell tumour was seen with cells having moderate to severe pleomorphism and mitosis of $>10 / 10 \mathrm{hpf}$, and extensive areas of necrosis (Figure 1a) (Figure 1b).

Impression of grade 3 sarcoma $(\mathrm{T} . \mathrm{D}=3$, mitosis $=2$, necrosis $=2$ ) was made based on FNCLCC grading system. Diagnostic possibilities considered were:
i. Leiomyosarcoma.
ii. Monophasic synovial sarcoma.

iii. MPNST.

IHC was done, tumour cells showed positivity for S-100 (Figure 2), and negative for desmin, CD34, HMB-45, CD99 and CK which was consistent with the diagnosis of MPNST. The patient received two cycles of post-operative chemotherapy (Ifosfamide+adriamycin) but expired eight months after diagnosis.

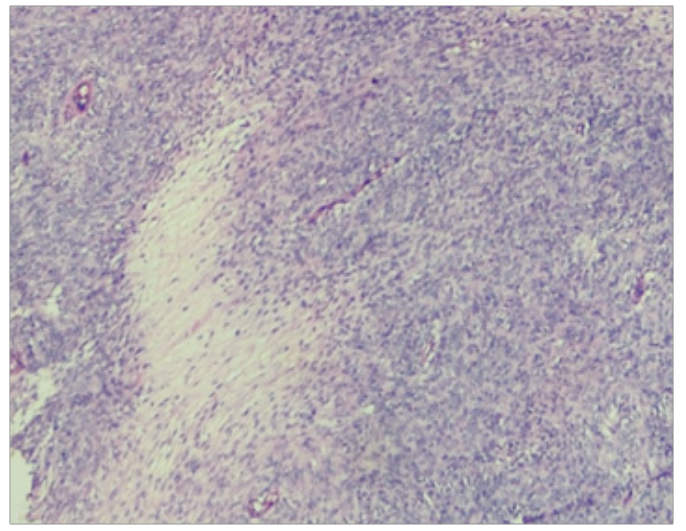

Figure I a Tumour showing areas of geographical necrosis with surrounding tumour cells at low power magnification.

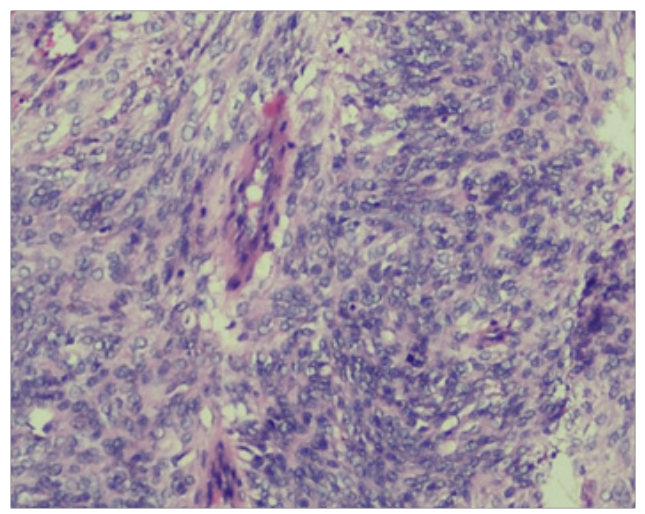

Figure Ib Higher magnification showing highly pleomorphic tumour cells (epitheloid type) arranged vessel with few mitotic figures. 


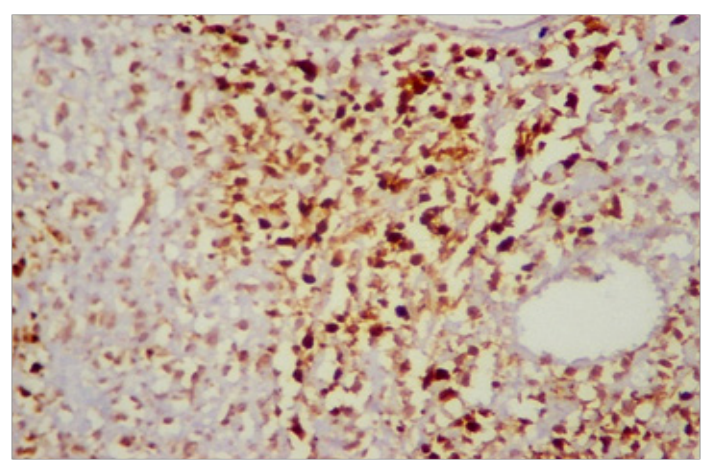

Figure 2 Tumour cells showing strong positivity for S-100.

\section{Discussion}

Primary pulmonary sarcomas (PS) comprise less than $1 \%$ of primary lung tumours. They represent $40 \%$ of rare pulmonary tumours and about $9 \%$ of all soft tissue sarcomas. ${ }^{6}$ MPNST is one of the rare soft tissue tumours comprising about $5-10 \%$ of all soft tissue sarcomas. ${ }^{7,8}$ These tumours are difficult and often create problem in diagnosis because of their histological similarities with other spindle cell sarcomas. Histological mimics include; leiomyosarcoma, monophasic synovial sarcoma, and fibro sarcoma. ${ }^{9}$ MPNST can arise from branches of major or minor peripheral nerves or their sheath. ${ }^{10}$ They can arise either spontaneously, or may have an association with neurofibromatosis type-1 (NF-1) in about $5-42 \%$ of adult cases. Although aggressive in nature, still surgery remains mainstay of treatment for MPNST lung. ${ }^{9}$

It has been reported that, 5-year survival rate may vary from $15-40 \%$. In patients where disease is associated with $\mathrm{NF}-1$ prognosis is generally poor. Associated adverse prognostic indicators include tumour size $>5 \mathrm{~cm}$, mitotic rate $>20 / 10 \mathrm{hpf}$, central location of tumour and incomplete resection. ${ }^{11}$ For diagnosing this tumour combination of gross, tumour histology and immunohistochemistry should be used.' Immunohistochemically tumour cells show reactivity for S-100 protein and Leu-7, in approximately half of the cases. ${ }^{12} \mathrm{~S}-100$ tends to be focal and not particularly strong, except in epitheloid variant of this tumour. ${ }^{13}$ Differential diagnosis of primary pulmonary MPNST include metastasis of an extra thoracic mesenchymal tumour into lung. The distinction of pulmonary MPNST from other primary lung sarcomas especially malignant mesenchymal tumours (like synovial sarcoma and leiomyosarcoma) is very difficult but crucial as the treatment options and prognosis differ. ${ }^{14}$

\section{Acknowledgements}

None.

\section{Conflict of interest}

Authors declare there is no conflict of interest.

\section{References}

1. Muwakkit SA, Rodriguez Galindo C, El Samra AI, et al. Primary malignant peripheral nerve sheath tumor of the lung in a young child without neurofibromatosis type1. Pediatr Blood Cancer. 2006;47(5):636-638.

2. Stephen R Grobmyer, John D Reith, Amir Shahlaee, et al. Malignant peripheral nerve sheath tumor: Molecular pathogenesis and current management considerations. J Surg Oncol. 2008;97(4):340-349.

3. Carli M, Ferrari A, Mattke A, et al. Pediatric malignant peripheral nerve sheath tumor: the Italian and German soft tissue sarcoma cooperative group. J Clin Oncol. 2005;23(33):8422-8430.

4. Shimizu J, Arano Y, Murata T, et al. A case of intrathoracic giant malignant peripheral nerve sheath tumor in neurofibromatosis type I. Ann Thorac Cardiovasc Surg. 2008;14(1):42-47.

5. ChaoBH, Stogner-Underwood KA, Kiev J, et al, Intrathoracic malignant peripheral nerve sheath tumor in neurofibromatosis 1. J Clin Oncol. 2008;26(13):2216-2218.

6. Etienne-Mastroianni B, Falchero L, Chalabreysse L, et al. Primary sarcomas of the lung: a clinicopathologic study of 12 cases. Lung Cancer. 2002;38(3):283-289.

7. Demir HA, Varan A, Yalcin B, et al. Malignant peripheral nerve sheath tumors in childhood,13 cases from a single centre. $J$ Pediatr Hematol Oncol. 2012;34(3):204-207.

8. Ferrari A, Bisogno G, Carli M. Management of childhood malignant peripheral nerve sheath tumor. Pediatric drugs. 2007;9(4):239-248.

9. Ducatman SB, Bernd WS, David GP. Malignant peripheral nerve sheath tumors- a clinicopathological study of 120 cases. Cancer. 986;57(10):2006-2021.

10. Hirose T, Scheitheuer BW, Sano T. Perineural malignant peripheral nerve sheath tumor - A clinico-pathologic, immune-histochemical and ultrastructural study of 7 cases. Am j surg pathol. 1998;22(11):1368-1378.

11. Coindre J M, Terrier P, Bui N B. Prognostic factors in adult patients with locally controlled soft tissue sarcoma- A study of 546 patients from the French federation of cancer centers sarcoma group. J clin Oncol. 1996;14(3):869-877.

12. Gray MH, Rosenberg AE, Dickersin GR, et al. GFAP and Keratin expression by benign and malignant peripheral nerve sheath tumor. Hum pathol. 1989;20(11):1089-1096.

13. Zhou H, Coffin CM, Perkins SL. Malignant peripheral nerve sheath tumor-A comparison of grade, immunophenotype and cell cycle/ growth activation marker expression in sporadic and neurofibromatosis-1 related lesions. Am j Surg Pathol. 2003;27(10):1337-1345.

14. Elvira LM, Renato F, Monica C. Primary intrapulmonary malignant peripheral nerve sheath tumor mimicking lung cancer. $J$ Thoracic Dis. 2013;5(4):E155-E157. 